\title{
Cardiopatías congénitas más frecuentes en niños con síndrome de Down
}

\author{
Miguel A. Ruz-Montes ${ }^{a}$, Eliana M. Cañas-Arenas ${ }^{\mathrm{b}}$, María A. Lugo-Posada ${ }^{\mathrm{c}, *}$, \\ María A. Mejía-Carmona ${ }^{c}$, Manuela Zapata-Arismendy ${ }^{c}$, Laura Ortiz-Suárez ${ }^{c}$ \\ y María I. Henao-Montaño ${ }^{c}$
}

\author{
a Cardiología, Clinica CardioVID, Medellín, Colombia \\ b Epidemiologia, Clinica CardioVID, Medellín, Colombia \\ c Pregrado de Medicina, Universidad Pontificia Bolivariana, Medellín, Colombia
}

Recibido el 25 de septiembre de 2015; aceptado el 9 de junio de 2016

Disponible en Internet el 21 de septiembre de 2016

\author{
PALABRAS CLAVE \\ Cardiopatía \\ congénita; \\ Comunicación \\ interventricular; \\ Hipertensión arterial \\ pulmonar; \\ Falla cardiaca
}

\begin{abstract}
Resumen
Introducción: El síndrome de Down es la alteración cromosómica más común entre los seres humanos, y está asociado, en su mayoría, a cardiopatías congénitas, principales causantes de mortalidad en los dos primeros años de vida.

Objetivo: Describir la frecuencia de cada cardiopatía congénita y sus características clínicas en niños con síndrome de Down atendidos en la Clínica Cardio VID de Medellín.

Metodología: Estudio observacional, descriptivo, de corte transversal, llevado a cabo en la Clínica Cardio VID, en pacientes con diagnóstico de síndrome de Down y cardiopatías congénitas desde el $1^{\circ}$ de enero de 2010 hasta el 31 de diciembre de 2013.

Resultados: Se seleccionaron 99 pacientes que cumplían con los criterios de inclusión, con edad promedio de 8,1 meses, 52 (52,5\%) mujeres. Según las historias clínicas, las cardiopatías congénitas más comunes en los niños con síndrome de Down fueron: comunicación interventricular perimembranosa en 61 casos $(61,6 \%)$, comunicación interauricular en $46(46,5 \%)$ y ductus arterioso en $38(38,4 \%)$.

Conclusiones: Por lo que se sabe, es el primer estudio epidemiológico en relación con la frecuencia y el tipo de cardiopatía congénita que se encuentra en los niños colombianos con síndrome de Down en un centro cardiovascular. La alta frecuencia de comunicación interventricular y la distribución diferencial de las malformaciones cardiacas asociadas con el síndrome de Down en la población, difieren de lo que se ha reportado en otros estudios.

(C) 2016 Sociedad Colombiana de Cardiología y Cirugía Cardiovascular. Publicado por Elsevier España, S.L.U. Este es un artículo Open Access bajo la licencia CC BY-NC-ND (http:// creativecommons.org/licenses/by-nc-nd/4.0/).
\end{abstract}

\footnotetext{
* Autor para correspondencia.

Correo electrónico: ma_alejalp@hotmail.com (M.A. Lugo-Posada).
} 


\section{KEYWORDS}

Congenital heart disease;

Ventricular septal defect;

Pulmonary arterial

hypertension;

Heart failure

\section{More frequent congenital cardiopathies in children with Down syndrome}

\begin{abstract}
Introduction: Down's syndrome is most common chromosomal alteration amongst humans, and it is mostly associated to congenital heart diseases, the main cause of mortality during the first years of life.

Motivation: To describe the frequency of each congenial heart disease and their clinical characteristics in children with Down's syndrome at the Cardio VID Clinic in Medellín.

Methods: Observational, cross-sectional, descriptive study conducted in the Cardio VID Clinic with patients diagnosed with Down's syndrome and congenital heart diseases between January $1^{\text {st }} 2010$ and December $31^{\text {st }} 2013$.

Results: 99 patients meeting all selection criteria were selected, with an average age of 8.1 months, 52 (52.5\%) female. According to their medical records, the most common congenital heart diseases in children with Down's syndrome were perimembranous ventricular septal defect in 61 cases (61.6\%), atrial septal defect in 46 (46.5\%) and ductus arteriosus in $38(38.4 \%)$.

Conclusion: To our knowledge it is the first epidemiological study regarding the frequency and type of congenital heart diseases found in Colombian children with Down's syndrome in a cardiovascular centre. The high frequency of ventricular septal defect and differential distribution of heart defects associated with Down's syndrome in the population differ to the data reported in other studies.

(c) 2016 Sociedad Colombiana de Cardiología y Cirugía Cardiovascular. Published by Elsevier España, S.L.U. This is an open access article under the CC BY-NC-ND license (http:// creativecommons.org/licenses/by-nc-nd/4.0/).
\end{abstract}

\section{Introducción}

El síndrome de Down se caracteriza por la trisomía completa del cromosoma 21 en $95 \%$ de los casos y ocurre aproximadamente en uno de cada 680 nacimientos vivos ${ }^{1}$. La mayoría de niños que nace con este síndrome padece malformaciones cardiacas en un 40 a 50\%, siendo una de las principales causas de morbimortalidad, especialmente en los primeros dos años; por tanto deben ser intervenidos cuanto antes a fin de optimizar su función cardiaca y mejorar el pronóstico de vida ${ }^{1,2}$.

La anomalía más común es el canal atrio-ventricular completo o el defecto total del septum atrio-ventricular que es casi exclusivo de estos pacientes y representa hasta el $80 \%$ de todos los casos diagnosticados. Otras anomalías son: comunicación interauricular, comunicación interventricular, persistencia del conducto arterioso y tetralogía de Fallot $^{2}$. En esta serie, el diagnóstico se realizó por ecocardiografía transtorácica en el $100 \%$ de los casos; ninguno fue llevado a cateterismo ni se utilizó otro tipo de imagen diagnóstica.

Estas cardiopatías tienen diferentes presentaciones clínicas y evolución. Los síntomas o signos de estas pueden estar ausentes en los primeros días, lo que conduce a un diagnóstico tardío. Esto puede ser determinante en el desarrollo de complicaciones como insuficiencia cardiaca, neumonía, arritmias cardiacas o hipertensión pulmonar ${ }^{2}$. Su tratamiento varía desde la resolución espontánea y la medicación, hasta una o más cirugías cardiacas.

Por tanto, teniendo en cuenta la importancia de las cardiopatías congénitas en los niños con síndrome de Down, este estudio trata de determinar la frecuencia y el perfil clínico de estas enfermedades en los pacientes atendidos en la Clínica Cardio VID, así como su presentación clínica, complicaciones y antecedentes perinatales, en busca de mejores programas de prevención, intervención y diagnóstico temprano, que disminuyan las complicaciones y mejoren la calidad de vida.

\section{Metodología}

Se realizó un estudio observacional descriptivo, de corte transversal, en el que se evaluaron niños con síndrome de Down que tenían cardiopatías congénitas y fueron atendidos en la Clínica Cardio VID de Medellín, del $1^{\circ}$ de enero de 2010 al 31 de diciembre de 2013. Se incluyeron desde neonatos hasta preescolares ( 0 a 6 años) con síndrome de Down y diagnóstico de cardiopatía congénita. Se excluyeron pacientes con antecedentes perinatales de síndrome de TORCH durante el embarazo y niños de madre con cardiopatía.

Para el procesamiento de esta información se construyó una base de datos en la cual se estandarizó el registro de las variables por cada uno de los investigadores, quienes fueron los encargados de descargar los datos.

En el plan de análisis se realizó la exploración de las variables, describiéndolas, ya fueran cuantitativas, mediante la utilización de medidas de tendencia central y de dispersión, o cualitativas mediante la codificación y determinación de la frecuencia absoluta y relativa. Así mismo, el análisis de la información recolectada se hizo en el programa estadístico SPSS, versión 2.0

\section{Resultados}

Se analizaron 99 pacientes con diagnóstico de síndrome de Down y cardiopatía congénita entre los años 2010 y 2013, de los cuales 47 eran de sexo masculino y 52 femenino; 
Tabla 1 Frecuencia de cardiopatías congénitas en niños con síndrome de Down en la Clínica Cardio VID en los años 2010-2013

\begin{tabular}{lll}
\hline Cardiopatía congénita & $\mathrm{n}=99$ & $\%=100 \%$ \\
\hline $\begin{array}{l}\text { Comunicación interventricular } \\
\quad \text { perimembranosa }\end{array}$ & 61 & 61,62 \\
Comunicación interauricular & 46 & 46,46 \\
Ductus arterioso & 38 & 38,38 \\
Canal aurículo-ventricular & 31 & 31,31 \\
Foramen oval permeable & 29 & 29,29 \\
Tetralogía de Fallot & 17 & 17,17 \\
Atresia pulmonar & 1 & 1,01 \\
Dos o más concomitantes & 72 & 72,73 \\
\hline
\end{tabular}

la cardiopatía congénita más prevalente fue la comunicación interventricular perimembranosa con $61,62 \%$. En la tabla 1 se hace referencia, en orden de frecuencia de mayor a menor, a los tipos de cardiopatías encontradas. Así mismo, se analizó el tipo de canal atrioventicular según la clasificación de Rastelli y se determinó que la más común es el tipo c. Además, se incluyó la presentación clínica, en la que el signo más presente fue el soplo cardiaco, en un $96 \%$, siendo el soplo holosistólico el más frecuente secundario a insuficiencia mitral. En la tabla 2 se enumeran los diferentes signos y síntomas encontrados. Adicionalmente, se estudiaron los factores asociados entre los cuales el más prevalente fue la hipertensión pulmonar con un $69,7 \%$; también se encontraron complicaciones asociadas a las cardiopatías (tabla 3). El hipotiroidismo fue la enfermedad más frecuente asociada con una prevalencia del $19 \%$. Los antecedentes perinatales hallados en las madres fueron diabetes mellitus gestacional $(8,1 \%)$ seguido de hipertensión arterial (4\%) e hipotiroidismo (2\%).

Tabla 2 Frecuencia de signos y síntomas en niños con síndrome de Down en la Clínica Cardio VID en los años 2010-2013

\begin{tabular}{lcc}
\hline Signos y síntomas & $\mathrm{n}=99$ & $\%=100 \%$ \\
\hline Soplo & 95 & 95,96 \\
Disnea & 38 & 38,38 \\
Cianosis & 22 & 22,22 \\
Arritmia & 2 & 2,02 \\
Ortopnea & 1 & 1,01 \\
Otros & 56 & 56,56 \\
\hline
\end{tabular}

Tabla 3 Frecuencias de factores asociados en niños con síndrome de Down en la Clínica Cardio VID en los años 20102013

\begin{tabular}{lcc}
\hline Factores asociados & $\mathrm{n}=99$ & $\%=100 \%$ \\
\hline Hipertensión pulmonar & 69 & 69,7 \\
Insuficiencia mitral & 22 & 22,22 \\
Infección respiratoria & 22 & 22,22 \\
Falla cardiaca & 16 & 16,2 \\
Edema pulmonar & 5 & 5,05 \\
Shock & 3 & 3,03 \\
Otras & 85 & 85,86 \\
\hline
\end{tabular}

La manifestación clínica más sobresaliente fue el soplo cardiaco (96\%), y de este el tipo que más se presentó fue el holosistólico $(44,4 \%)$; como síntomas estuvieron la disnea $(38,4 \%)$ y otros $(56,6 \%)$, dentro de estos la diaforesis $(5,1 \%)$. Otros estudios reportan que las manifestaciones clínicas más prevalentes son los pacientes asintomáticos (36\%), seguido por insuficiencia cardiaca $(33 \%)$, disnea $(15 \%)$, cianosishipoxia (10\%) y soplo cardiaco $(4 \%)^{3-5}$, que por el contrario en los resultados de este estudio fue el más habitual. El conocimiento de los signos y síntomas más frecuentes sumado al uso apropiado de las técnicas de diagnóstico y de tratamiento, es crucial para asegurar una terapéutica óptima y efectiva de los pacientes con cardiopatía, así como para conseguir una relación costo-beneficio adecuada, según las guías españolas de cardiopatía congénita ${ }^{5}$.

\section{Discusión}

El síndrome de Down o trisomía 21, se ha asociado con diversas cardiopatías congénitas como defectos del canal atrio-ventricular, comunicación interventricular e interauricular, tetralogía de Fallot o prolapso de válvular mitral ${ }^{1}$. En el análisis de los pacientes con defectos del canal atrioventricular se tuvo en cuenta la clasificación de Rastelli, en la que el canal se divide en tres tipos ( $A, B$ y $C$ ) de acuerdo con la inserción de las valvas que conforman la válvula atrio-ventricular, con base en la relación de las valvas anteriores (puente superior y anteroseptal), a la cresta del septo intervenventricular (SIV). El más frecuente es el tipo A (50\%) en cuyo caso el velo puente superior está íntimamente relacionado con la cresta; en general, se asocia con síndrome de Down. En el tipo B (el menos frecuente, 3\%), la inserción se realiza a través de un músculo papilar de la región derecha SIV y casi siempre se asocia con un defecto septal atrio-ventricular disbalanceado de predominio derecho. En el tipo C (30\%) el velo puente superior es de gran tamaño, no se inserta en el septo interventricular (velo flotante) pero sí en un músculo papilar anterior. Este tipo suele observarse en la heterotaxia visceral y en las malformaciones cono-truncales ${ }^{6}$. En la investigación, la cardiopatía congénita más habitual fue la comunicación interventricular perimembranosa, lo cual muestra la prevalencia más alta del total analizado con 61,62\%. De igual manera, en varios estudios la alteración anatómica congénita más representativa en los niños con síndrome de Down es la comunicación interventricular perimembranosa, que representa del $15 \mathrm{al}$ $20 \%$ de todas las cardiopatías congénitas, con una incidencia de 6-10 por cada 1.000 o 20-30 por cada 10.000 nacimientos, seguida por el canal aurículo-ventricular, el ducto arterioso, la comunicación interauricular y la tetralogía de Fallot ${ }^{3}$, a diferencia de otros estudios hechos en Brasil y España que arrojaron como cardiopatía más frecuente el defecto del septo aurículo-ventricular, con una prevalencia de 36,3\% y $89 \%$, respectivamente $e^{4,5,7}$.

En las 99 historias clínicas analizadas se encontró que el sexo femenino, con 52 pacientes, predominó sobre la población masculina, 47 pacientes, y que la edad promedio en meses al momento del diagnóstico fue de 8.1 meses (0-72 meses). Se evidenció, así mismo, un retardo en el diagnóstico de las cardiopatías congénitas, lo que puede explicarse porque la trisomía 21 no siempre se detecta de 
manera prenatal o al momento del nacimiento, de acuerdo con un estudio llevado a cabo en Brasil en una unidad materno infantil de un centro de referencia de la localidad, en el cual se documentó que sólo el $74 \%$ de los casos se descubrió dentro de los seis primeros meses de edad ${ }^{7}$.

La asociación más frecuente fue la hipertensión pulmonar, dada probablemente por el aumento del flujo sanguíneo pulmonar, teoría apoyada por los resultados arrojados en un estudio realizado a niños con cardiopatía congénita asociada a síndrome de Down en el Instituto Nacional de Pediatría de México, en el cual se reportó que el $50 \%$ de los casos se asociaba a dicha condición ${ }^{7,8}$. Un gran porcentaje de niños con síndrome de Down tiene asociada una cardiopatía congénita, con lo cual se demuestra la importancia del diagnóstico y el tratamiento oportunos de esta patología, que como se pudo evidenciar, trae consigo complicaciones y enfermedades asociadas que pueden poner en riesgo la vida de los niños, especialmente en sus primeros dos años de vida ${ }^{8,9}$.

En cuanto a antecedentes familiares se describen la edad materna, con un promedio de 32,68 años (15-45 años), y la paterna de 35,73 años (18-54 años), datos que concuerdan con otros estudios en los que se evidencia que la mayor prevalencia de síndrome de Down se da en madres mayores de 35 años $^{7,9}$ y padres mayores de $40^{9}$, a excepción de un trabajo realizado en México en el cual ocurría con mayor frecuencia en madres de 16 a 25 años $^{4}$. El antecedente patológico materno más importante fue la diabetes mellitus gestacional $(8,1 \%)$, seguido por la hipertensión arterial crónica (4\%). En cuanto a la edad materna, es posible concluir que hay una variabilidad en el rango, llegando a edades mayores de 35 años, que se conoce como un factor de riesgo importante para el desarrollo de síndrome de Down, de modo que la consejería antes de la concepción puede ser indispensable en la prevención primaria. En el mundo, el síndrome de Down es la principal causa genética de cardiopatías congénitas ${ }^{9,10}$; entre 40 a $60 \%$ de los recién nacidos se ven afectados por defectos congénitos del corazón ${ }^{10,11}$.

Es importante resaltar que la patología más asociada entre los niños fue el hipotiroidismo, que puede estar más relacionado con la alteración cromosómica, pues se tiene evidencia de que el síndrome de Down presenta más de 80 manifestaciones clínicas sistémicas asociadas como: enfermedad cardiaca congénita, estenosis o atresia duodenal, ano imperforado, enfermedad de Hirschsprung, hipotonía muscular, deficiencias del sistema inmunológico, mayor riesgo de leucemia en la infancia, enfermedad de Alzheimer de inicio temprano ${ }^{12}$, patología ocular, hipoacusia y patología tiroidea, además de mayor frecuencia de enfermedades infecciosas, especialmente respiratorias ${ }^{13}$.

\section{Conclusiones}

En niños con síndrome de Down, el examen físico adecuado y la educación a los padres para la vigilancia de la aparición de las manifestaciones más frecuentes previamente descritas, pueden contribuir al diagnóstico y la intervención precoces. En cuanto a los factores asociados, es imperativo que el personal de la salud los identifique ya que dada su frecuencia, deben estar preparados para ofrecer un tratamiento oportuno en el centro de salud y evitar las complicaciones inherentes a la cardiopatía. En vista de que el antecedente patológico materno más frecuente fue la diabetes mellitus gestacional, se puede confirmar la relevancia de los controles prenatales y del seguimiento a estas madres con este antecedente para evitar complicaciones como las cardiopatías congénitas.

\section{Limitaciones}

En el estudio se presentaron problemas en la recolección de la información por falta de información en la historia clínica y no fue posible contactar a los padres o responsables por el cambio de datos personales.

Una de las principales limitaciones fue el tamaño reducido de la muestra y que el estudio se hiciera en un centro cardiovascular donde solo acude población pediátrica con cardiopatía congénita, lo cual limita información clave para conocer la prevalencia de las cardiopatías congénitas en la población general con síndrome de Down. También se tuvo en cuenta que en la ciudad no se han desarrollado estudios de este tipo, por lo que se considera una investigación importante, pues se podrían realizar otros estudios basados en los datos epidemiológicos que se encontraron e incluir mayor cantidad de pacientes y por ende más instituciones, y así poder hacer comparaciones más amplias y reveladoras.

Estas limitaciones nos han llevado a subestimar la prevalencia de malformaciones cardiacas congénitas en nuestros niños con síndrome de Down, y también podría explicar las diferencias en la prevalencia de los defectos encontrados en este estudio. A pesar de estas limitaciones, creemos que hemos hecho algunos progresos. La mayoría de nuestros pacientes son diagnosticados y ahora se someten a una cirugía en el primer año de vida.

\section{Responsabilidades éticas}

Protección de personas y animales. Los autores declaran que para esta investigación no se han realizado experimentos en seres humanos ni en animales.

Confidencialidad de los datos. Los autores declaran que en este artículo no aparecen datos de pacientes.

Derecho a la privacidad y consentimiento informado. Los autores declaran que en este artículo no aparecen datos de pacientes.

\section{Conflicto de intereses}

Los autores declaran no tener ningún conflicto de intereses.

\section{Agradecimientos}

A la Clínica Cardio VID por facilitar el acceso a las historias clínicas.

\section{Bibliografía}

1. Aracena M. Cardiopatías congénitas y síndromes malformativos genéticos. Rev Chil Pediatr. 2003;74:426-31. 
2. Nuñez Gomez F, Lopez-Prats, Lucea JL. Cardiopatías congénitas en niños con síndrome de Down. Rev Esp Pediatr. 2012;68:415-20.

3. Figueroa J, Pozzo B, Pablos J, Calderon C, Castrejón R. Malformationes cardíacas en los niños con síndrome de Down. Rev Esp Cardiol. 2003;56:894-9.

4. Alves F, Riberta L, da Silva S. Prevalencia y perfil de las cardiopatías congénitas e hipertensión pulmonar en el síndrome de Down en el servicio de cardiología pediátrica. Rev Paul Pediatr. 2014;32:159-63.

5. Martínez E, Rodríguez F, Medina J. Evolución clínica en pacientes con síndrome de Down y cardiopatía congénita. Cir Cir. 2010;78:245-50.

6. Vázquez Antona CA, Muñoz Castellanos L, Roldán FJ, Erdmenger Orellana J, Romero Cárdenas A, Vargas Barrón J. Espectro anatómico entre el defecto de la tabicación atrioventricular completo y parcial. Evaluación con ecocardiografía bi y tridimensional. Arch Cardiol Méx. 2008;78:40-51.

7. Vilas Boas LT, Albernaz EP, Gonçalves Costa R. Prevalence of congenital heart defects in patients with Down syndrome in the municipality of Pelotas, Brazil. J Pediatr. 2009;85: 403-7.

8. Maroto Monedero C, Enríquez de Salamanca F, Herráiz Sarachaga I, Zabala Argüelles JI. Guías de práctica clínica de la Sociedad Española de Cardiología en las cardiopatías congénitas más frecuentes. Rev Esp Cardiol. 2010;54:67-82.

9. Nahar R, Kotecha U, Dua Puri R, Mohan Pandey R, Chander Verma I. Survival analysis of Down syndrome cohort in a tertiary health care center in India. Indian J Pediatr. 2012:105-10.

10. Eyzaguirre F, Unanue N, Mericq V. Hipertiroidismo y síndrome de Down: caso clínico. Rev Chil Pediatr. 2008;79: 290-4.

11. Nazer J, Cifuentes L. Estudio epidemiológico global del síndrome de Down. Rev Chil Pediatr. 2011;82:105-12.

12. Sommer C, Henrique-Silva F. Trisomy 21 and Down syndrome: a short review. Braz J Biology. 2008;68:447-52.

13. Retamales M, Moreno R, González A, Cerda LJ, Lizama CM. Morbilidad y mortalidad durante el primer año de vida en pacientes con síndrome de Down. Rev Chil Pediatr. 2009;80: 323-31. 